\title{
Síndrome de Down: epidemiologia e alterações oftalmológicas
}

\section{Down syndrome: epidemiology and ophthalmologic changes}

Silvia Helena Tavares Lorena ${ }^{1}$

\begin{abstract}
Resumo
Objetivo: O objetivo do projeto de pesquisa é determinar a incidência das alterações oftalmológicas de crianças com a Síndrome de Down, as quais foram atendidas no Ambulatório de Especialidades Jardim Peri-Peri, no período de 7 de julho 2004 a 14 de outubro de 2009. Métodos: Neste trabalho empregou-se um estudo observacional retrospectivo dos prontuários de 35 crianças com Síndrome de Down, 15 do sexo feminino e 20 do masculino, da raça branca e com 2 meses de idade. Os pacientes, no período em estudo, foram submetidos à avaliação oftalmológica que incluiu: -inspeção ocular; retinoscopia; biomicroscopia; mapeamento de Retina; teste de Milder; teste de observação da fluoresceína na orofaringe (TOFO). Resultados: As crianças deste estudo foram examinadas desde 2 meses de idade e acompanhadas anualmente. As alterações oftalmológicas encontradas nas 35 crianças com a Síndrome de Down foram: fissura palpebral oblíqua em 100\%, epicanto em $70 \%$, vasos supranumerários nas arcadas ao exame de mapeamento de retina em $100 \%$,manchas de Brushfield em $48,57 \%$ ,blefarite em $42,85 \%$, obstrução da via lacrimal excretora em $25,71 \%$, miopia em $14,28 \%$, astigmatismo em $14,28 \%$, ambliopia em 2,85\%, estrabismo convergente em $11,42 \%$, estrabismo divergente em 5,71\%, nistagmo em 2,85\%, ectrópio em 5,71\%, catarata congênita em 2,85\%. Conclusão: Neste trabalho verificou-se a alta incidência de patologias oftalmológicas em crianças com a síndrome de Down, tais como: blefarites, obstrução da via lacrimal excretora, erros refracionais, estrabismos e catarata etc. As alterações oftalmológicas são muito importantes para alertar os pais das crianças com síndrome de Down, quanto ao seu tratamento precoce proporcionando melhora na acuidade visual e no desenvolvimento neuropsicomotor dessas crianças.
\end{abstract}

Descritores: Síndrome de Down/complicações; Oftalmopatias/epidemiologia; Anormalidades do olho

\begin{abstract}
Purpose: The research project goal is to determine the incidence of ophthalmologic changes in children with Down syndrome who were attended in Peri-Peri Health Center, from july 07, 2004 to october 14, 2009. Methods: This is an observational retrospective study of 35 children medical records with Down's Syndrome who were attended in Peri-Peri Health Center. The patients were submitted to ophthalmologic exams including: ocular inspection; retinoscopy; biomicroscopy; indirect ophthalmoscopy; fluorescein disappearance test; observation test of fluorescein in the oropharynx. Results: The children of this study were examined since 2 months of age. The ophthalmologic changes found in the 35 children with Down Syndrome was: oblique eyelid in $100 \%$, epicanto in $70 \%$, supernumerary vessels in the arcades in the retina exam in $100 \%$, Brushfield spot in $48.57 \%$, blefarity in $42.85 \%$, congenital lacrimal obstruction in $25.71 \%$, miopy in $14.28 \%$, astigmatismy in $22.28 \%$, esotropia in $11.42 \%$, exotropia in $5.71 \%$, nystagmus in $2.85 \%$, ectropion in $5.71 \%$ and congenital cataract in $2.85 \%$. Conclusion: This study found a lot of ophthalmologic diseases in children with Down's syndrome: blefarity, congenital nasolacrimal duct obstruction, etc. Therefore the follow-up of these children is very relevant.
\end{abstract}

Keywords: Down syndrome/physiopathology; Eye diseases/epidemiology; Eye abnormalities

${ }^{1}$ Ambulatório Municipal de Especialidades Jardim Peri-Peri. São Paulo (SP), Brasil

Trabalho realizado no Ambulatório Municipal de Especialidades Jardim Peri-Peri - São Paulo (SP), Brasil.

Os autores declaram inexistir conflitos de interesse

Recebido para publicação em 30/11/2010 - Aceito para publicação em 27/6/2011

Rev Bras Oftalmol. 2012; 71 (3): 188-90 


\section{INTRODUÇÃO}

$\Delta$ Síndrome de Down foi descrita em 1866 por um médico britânico: John Langdon Down. A síndrome é também denominada de Trissomia do Cromossomo 21.(1)

O diagnóstico desta síndrome é realizado através da cariotipagem cromossômica, que pode ser realizada durante a gestação, por análise citogenética das vilosidades coriônicas ou células do líquido amniótico. ${ }^{(2-4)}$

No Brasil a incidência desta síndrome é de 1,13:1000 nascidos vivos. $^{(5)}$

$\mathrm{O}$ aconselhamento se faz mister, pois pais de crianças com a Síndrome de Down, têm risco aumentado desta síndrome na prole futura. ${ }^{(6-9)}$

As alterações oftalmológicas encontradas na Síndrome de Down por ordem de frequência são: fendas palpebrais estreitas e oblíquas, epicanto, ametropias (alta miopia), anomalias de íris (manchas de Brushfield), estrabismo, blefarite, obstrução das vias lacrimais excretoras, alterações retinianas, ambliopia, nistagmo, catarata, ceratocone, eversão congênita das pálpebras superiores, ectrópio ou entrópio das pálpebras, euribléfaro. $^{(8-14)}$

O objetivo do projeto de pesquisa é determinar a incidência das alterações oftalmológicas de crianças com a Síndrome de Down, atendidas no Ambulatório de Especialidades Jardim Peri-Peri, no período de 7 de julho 2004 a 14 de outubro de 2009.

\section{Métodos}

Estudo observacional retrospectivo dos prontuários de 35 crianças, 15 do sexo feminino e 20 do sexo masculino com Síndrome de Down, todos da raça branca e com 2 meses de idade, atendidos no Ambulatório de Especialidades Jardim Peri-Peri, no período de 7 de julho 2004 a 14 de outubro de 2009.

Os pacientes, no período em estudo, foram submetidos à avaliação oftalmológica que incluiu:

-Inspeção ocular;

-Retinoscopia;

-Biomicroscopia;

-Mapeamento de retina;

-Teste de Milder;

- Teste de observação da fluoresceína na orofaringe (TOFO).

A inspeção ocular foi realizada com a finalidade de diagnosticar: estrabismo, as más posições palpebrais (ectrópio, entrópio) e dacriocistite crônica.
A biomicroscopia foi realizada a fim de detectar alguma patologia externa ocular (blefarite, conjuntivite, etc).

A retinoscopia foi realizada com régua de esquiascopia sob dilatação com midriático, objetivando diagnosticar ametropias (miopia, astigmatismo, etc).

A oftalmoscopia binocular indireta foi realizada, após a dilatação das pupilas, utilizando-se colírios de tropicamida a $1 \%$ e fenilefrina a $2,5 \%$, uma gota em cada olho, com intervalo de 5 minutos, por 3 vezes, cerca de 40 minutos antes do exame. A oftalmoscopia binocular indireta foi realizada com lente de 20 dioptrias.

-Teste do desaparecimento da fluoresceína (Teste de Milder)

-Teste de observação da fluoresceína na orofaringe (TOFO)

\section{Resultados}

As alterações oftalmológicas encontradas nas 35 crianças com a Síndrome de Down foram: fissura palpebral oblíqua em $100 \%$, epicanto em $70 \%$, vasos supranumerários nas arcadas ao exame de mapeamento de retina em $100 \%$, blefarite em $42,85 \%$, obstrução da via lacrimal excretora em $25,71 \%$, miopia em $14,28 \%$, astigmatismo em $22,85 \%$, hipermetropia em $20 \%$,emétropes em $42,85 \%$, esotropia em $11,42 \%$, exotropia em $5,71 \%$, nistagmo em $2,85 \%$, ectrópio em $5,71 \%$, catarata congênita em $2,85 \%$, manchas de Brushfield em 48,57. A catarata congênita ocorreu em $2,85 \%$ das crianças examinadas.

Neste trabalho, a incidência de ectrópio correspondeu à $5,71 \%$ e a obstrução da via lacrimal excretora obteve uma incidência de $27,27 \%$.

As principais alterações oculares na síndrome de Down deste trabalho como: fissura oblíqua (35 crianças), obstrução lacrimal congênita (30 crianças), manchas de Brushfield (17 crianças), blefarite (15 crianças), miopia (5 crianças), esotropia (4 crianças) estão representadas no Gráfico 1.

\section{Dıscussão}

Segundo a literatura ${ }^{(10)}$ é grande a incidência de fissura palpebral oblíqua (98\%) e o epicanto convencional (61\%) em crianças com a Síndrome de Down, o que é também constatado com as crianças deste estudo. Os 
achados de blefarite em $42,85 \%$ das crianças examinadas corrobora com a literatura que mostra incidência em torno de $30 \%{ }^{\left({ }^{(8)}\right.}$

Nesta casuística, o tipo de estrabismo mais frequente foi a esotropia, o que condiz com a literatura. ${ }^{(8)}$

$\mathrm{O}$ vício refracional mais frequente, neste trabalho, foi o astigmatismo com $22,85 \%$, seguido da hipermetropia com $20 \%$ e miopia com $14,28 \%$, correspondendo com a literatura. ${ }^{(8)}$

Segundo a literatura ${ }^{(6,7,10)}$, os vasos supranumerários nas arcadas são patognomônico desta síndrome, o que condiz com os achados no mapeamento de retina das crianças deste trabalho.

A endostatina representa uma proteína codificada no cromossomo 21, sendo um potente inibidor da angiogênese, inibindo a proliferação das células endoteliais e de migração. A redução da angiogênese sistêmica por alto nível de endostatina em indivíduos com síndrome de Down são responsáveis pela ramificação de vasos da retina central.

A incidência de nistagmo na literatura ${ }^{(10)}$ corresponde a $6 \%$, outros estudos ${ }^{(8)}$ apontam $18 \%$. Neste presente estudo, a incidência de nistagmo correspondeu a $2,8 \%$, muito abaixo da porcentagem descrita na literatura devido ao menor número de indivíduos estudados.

As manchas de Brushfield foram observadas em $48,57 \%$ das crianças examinadas, embora a literatura afirme que as manchas desaparecem no primeiro ano de vida, a alta incidência é devido ao fato destas crianças terem sido examinadas a partir de 2 meses de idade. ${ }^{(8)}$

A catarata congênita ocorreu em $2,85 \%$ das crianças examinadas, porém segundo a literatura sua incidência é em torno de $13 \%$ das crianças, ocorrendo mais comumente em maiores de 12 anos. ${ }^{\left({ }^{(8)}\right.}$

Neste trabalho, a incidência de ectrópio correspondeu a $5,71 \%$, o que confirma os dados da literatura , a qual afirma que o ectrópio congênito é mais frequente em crianças com a Síndrome de Down devido à associação com a flacidez ligamentar. ${ }^{(12)}$

A obstrução da via lacrimal excretora obteve uma incidência de $27,27 \%$, correspondendo com os dados da literatura. ${ }^{(8)}$

\section{ReferêNCIAS}

1. Catalano RA. Down syndrome. Surv Ophthalmol. 1990;34(5):385-98. Review.

2. Cullen JF. Blindness in mongolism (Down's syndrome). Br J Ophthalmol. 1963;47:331-3.

3. Nussbaum RL, McInnes RR, Huntington FW. Thompson \& Thompson genética médica. 6a ed. Rio de Janeiro: Guanabara Koogan; 2002.
Gráfico1

\section{Principais alterações oftalmológicas na síndrome de Down encontradas neste trabalho}

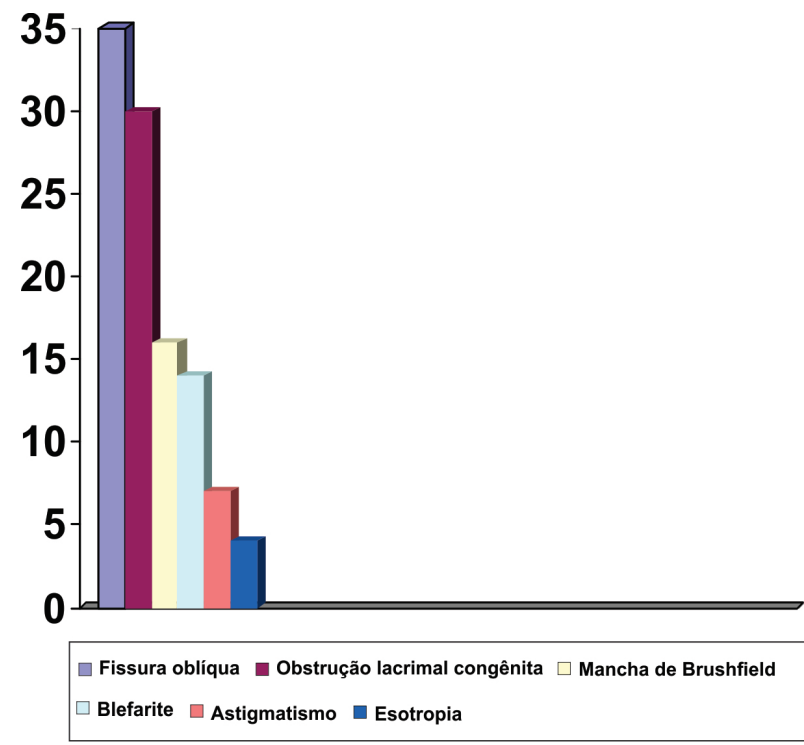

4. Smith DW. Síndromes de malformações congênitas: aspectos genéticos, embriológicos e clínicos. 3a ed. São Paulo: Manole; 1985.

5. Frota-Pessoa O, Otto PA, Otto PG. Mongolismo. In: FrotaPessoa O, Otto PA, Otto PG, editores. Genética clínica. São Paulo: Francisco Alves; 1987. p. 143-567.

6. Parsa CF, Almer Z. Supranumerary optic disc vessels may indicate reduced systemic angiogenesis in Down syndrome. Br J Ophthalmol. 2008;92(3):432-3.

7. Jaeger EA. Ocular findings in Down's syndrome. Trans Am Ophthalmol Soc. 1980;78:808-45.

8. Cunha RN, Moreira JB. Manifestações oculares em crianças e adolescentes com Síndrome de Down. Arq Bras Oftalmol. 1995;58(3):152-7

9. Matayoshi S, Sardinha M, Cozac AL, Araf D, Moura EM. Síndrome de Down e alterações de vias lacrimais. Arq Bras Oftalmol. 2003;66(4):481-4.

10. Fimiani F, Iovine A, Carelli R, Pansini M, Sebastio G, Magli A. Incidence of ocular pathologies in Italian children with Down syndrome. Eur J Ophthalmol. 2007;17(5):817-22.

11. Shapiro MB, France TD. The ocular features of Down's syndrome. Am J Ophthalmol. 1985;99(6):659-63.

12. Shiratori CA, Komatsu MC, Schellini SA, Cvintal T. Ectrópio palpebral em portador da síndrome de Down e conjuntivite alérgica: relato de caso. Arq Bras Oftalmol. 2007;70(4):706-8.

13. Kanski JJ. Oftalmologia clínica: uma abordagem sistemática. 5a. ed. Rio de janeiro: Elsevier; 2004.

14. Botelho PBM, Marback P, Sousa LB, Campos M, Vieira LA Ceratoconjuntivite alérgica e complicações do segmento ocular anterior de pacientes. Arq Bras Oftalmol. 2003;66(1):25-8.

\section{Endereço para correspondência}

Silvia Helena Tavares Lorena

Rua Flórida,1404 - Brooklin

CEP 04561-030 - São Paulo (SP), Brasil

Fone:(11) 5507-2705

E-mail: slorena1990@hotmail.com 